\title{
Civilisations
}

Revue internationale d'anthropologie et de sciences

humaines

59-2 | 2011

Les apparences de l'homme

\section{Généalogie de la parure}

Du blason comme modèle sémiotique au tissu comme modèle organique

\section{Thomas Golsenne}

\section{(2) OpenEdition}

1 Journals

\section{Édition électronique}

URL : http://journals.openedition.org/civilisations/2569

DOI : 10.4000/civilisations.2569

ISSN : 2032-0442

Éditeur

Institut de sociologie de l'Université Libre de Bruxelles

\section{Édition imprimée}

Date de publication : 30 juin 2011

Pagination : 41-58

ISBN : 2-87263-034-1

ISSN : 0009-8140

\section{Référence électronique}

Thomas Golsenne, «Généalogie de la parure », Civilisations [En ligne], 59-2 | 2011, mis en ligne le 04 juillet 2014, consulté le 19 avril 2019. URL : http://journals.openedition.org/civilisations/2569; DOI : $10.4000 /$ civilisations. 2569 


\title{
Généalogie de la parure \\ Du blason comme modèle sémiotique au tissu comme modèle organique
}

\author{
Thomas GOLSENNE
}

Résumé : L'idée que seul l'homme modifie son apparence corporelle paraît évidente ; elle s'appuie sur une conception naturaliste de l'humain, qui le distingue radicalement des autres animaux en tant qu'être de culture ; elle s'incarne par exemple, en histoire, dans la codification des apparences au Moyen Âge, et en ethnologie, dans la pensée de Lévi-Strauss. Elle est pourtant contredite par d'autres discours: l'anthropologie de Descola, qui par le biais de l'animisme montre que les animaux, aussi, portent des vêtements ; l'éthologie, capable de penser une sémiotique de la parure animale; et la morphogenèse, qui étudie la forme d'un point de vue dynamique. Ces discours sont tous traversés par la métaphore du blason: modèle efficace, mais limité, pour penser la signification de la parure, tant humaine qu'animale. Tout semble pourtant mener, dans ces remises en cause du modèle naturaliste, au dépassement de l'approche sémiotique et à une conception plus vitaliste de la parure.

Mots-clés : parure, vêtement, héraldique, anthropologie, éthologie, histoire, Moyen âge, morphogenèse, humanisme, esthétique.

Abstract: It seems obvious that only Man modifies his bodily appearance; this idea is based on a naturalistic conception of the human being; as a cultural being, he is radically distinguished from other animals. This conception is illustrated in history for instance, in the way appearances are codified in the Middle Ages, and in ethnology, in Lévi-Strauss's works. It is nevertheless contradicted by other theories: Descola's anthropology shows, through animism, that animals wear clothes, too; ethology is able to elaborate a semiotics of animal adornment; and morphogenesis studies forms from a dynamic point of view. All these theories are related to the metaphor of heraldry: it is an efficient-but incomplete-model allowing to define the signification of human as well as animal adornment. When putting at stake this naturalistic model, everything seems to lead to an overcoming of the semiotic point of view, towards a more vitalistic conception of adornment.

Keywords: adornment, clothing, heraldry, anthropology, ethology, history, Middle Ages, morphogenesis, humanism, aesthetics. 


\section{L}

'homme semble avoir modifié son apparence corporelle depuis la nuit des temps ; seul l'homme modifie son apparence corporelle - ce sont là des lieux communs corrélés qui font de la parure un de ces domaines spécifiquement réservés à l'être humain dans la pensée occidentale moderne. Gottfried Semper l'avait déjà très bien énoncé en 1869 :

Le fait d'orner est en effet un très étrange phénomène de l'histoire culturelle! Il appartient en propre à l'homme, et est peut-être la chose la plus ancienne dont il a fait usage. Aucun animal ne se pare. La corneille paradant avec des plumes d'autres oiseaux est comme on sait une chimère. (Semper 2007 : 345-46)

Ce qu'affirme-là Semper fait partie d'une série de textes d'ethnologues et d'historiens de l'art qui partagent l'idée que dès que l'homme fut homme, dès qu'il quitta la nature pour entrer dans la culture, si on veut dire les choses grossièrement, il modifia artificiellement son apparence ainsi que celle de son environnement : l'art (et sa première manifestation, la parure) n'aurait pas été autre chose qu'un marqueur d'humanité. Encore récemment, la préhistorienne Yvette Taborin maintenait que :

De l'élaboration d'une idée qui entre dans le système des valeurs du groupe, racontée et mille fois répétée, à sa communication grâce au code de la parure, il y a un progrès mental énorme : celui de la symbolisation d'un objet, qui perd partiellement sa propre identité pour être partie de l'idée. C'est le transfert d'un objet matériel, concret, dans la sphère intellectuelle des humains. Cet acquis signe la plénitude enfin acquise de l'espèce humaine. (Taborin 2004 : 10-11)

On ne cherchera pas dans cet article à répondre au premier degré à la question qui vient naturellement à l'esprit : pourquoi l'homme est-il vraiment humain quand il modifie son apparence corporelle. Car disons-le nettement : il n'y a pas de bonne réponse à une mauvaise question. On cherchera plutôt à comprendre d'abord quelle est l'image de l'humanité qui est induite par ce questionnement.

Dans une première approximation, il apparaitra vite que cette image découle de ce que Philippe Descola appelle l'ontologie naturaliste qui, pour aller vite, s'est élaborée en Europe depuis Aristote et est la base de la pensée moderne. Ontologie dans laquelle nature et animalité d'une part, culture et humanité d'autre part, sont séparées d'un même trait radical (Descola 2005 : 58-131, 241-79). Et, pour confirmer cette première observation, on s'intéressera au système de pensée que Descola présente comme l'inverse du naturalisme, à savoir l'animisme, dans lequel l'idée que seul l'homme peut modifier son apparence corporelle est une absurdité : les Indiens jivaros traiteraient Semper de fou.

Mais cette réponse ethnologique qui nous fournira une belle leçon de relativisme culturel ne va pas suffire ; car force est de constater que les Jivaros ne sont pas les seuls à penser la possibilité d'une parure animale: des biologistes et des éthologues, des scientifiques imprégnés de l'ontologie naturaliste, donc, remettent à leur tour fortement la conviction de la solitude ornementale de l'homme. On verra comment cette remise en question modifie presque tout le paysage conceptuel où prennent place l'humanité, l'animalité et la parure.

Presque, car demeure un point fixe, véritable pivot d'Archimède : l'approche sémiotique de la parure. Presque tous ceux qui se sont penchés sur la question de la parure, qui ont voulu lui donner du sens (quel que soit celui-ci) ont en commun l'idée que la parure fonctionne comme une sorte de langage codé. Il n'est pas étonnant, dès lors, de trouver, très fréquemment 
dans ces débats (comme on va s'en rendre compte bientôt), l'image du blason, le code visuel par excellence des apparences corporelles. L'héraldique médiévale semble fournir un modèle insurpassable pour penser la signification de la «parure », aussi bien humaine qu'animale. Cette obsession du sens et cette prégnance du modèle héraldique caractérisent la grande majorité des travaux scientifiques sur la parure et se présentent comme leur présupposé implicite. C'est donc là que la critique constructive doit viser en priorité si elle a pour objectif de débarrasser la réflexion scientifique de ses impensés.

\section{La parure inorganique, ou la fabrique de la culture}

L'idée que seul l'homme se construit une apparence artificielle est profondément ancrée dans l'histoire occidentale des idées. Pour aller vite, l'apparition du vêtement et de la parure comme problèmes en soi naît avec le christianisme. Gil Bartholeyns a bien montré que dans la culture gréco-latine, les apparences étaient soumises aux règles relatives de la décence, de la dignité humaine (Bartholeyns 2011). Mais les Pères de l'Église, interprétant la Genèse, vont plus loin : le vêtement est le signe de la nature déchue de l'homme, obligé, depuis le Péché Originel, de se recouvrir de «tuniques de peau » pour cacher ses pudenda. Ainsi Augustin distingue-t-il l'homme de la Nature (avant la Chute, c'est-à-dire créature ressemblante à Dieu) et l'homme de la nature postadamique (après la Chute), qui entre dans un monde forgé par lui, sa volonté et son libre arbitre : la culture (Bartholeyns 2009 : 102-104). Autrement dit l'homme chrétien doit retrouver la « nudité » originelle pour retrouver Dieu : « suivre nu le Christ nu », dit l'adage médiéval.

Mais, dans le même temps, émerge une anthropologie positive de la spécificité de l'homme au sein de la Création : se revêtant d'artifice, il n'est plus un animal. Les médiévaux retrouvaient ainsi parfois une idée antique : l'homme, le plus démuni des animaux, leur est supérieur parce qu'il compense ses faiblesses de départ par son invention et ses réalisations (Bartholeyns 2009: 112). Le vêtement acquiert ainsi une fonction humanisante: dans beaucoup de romans du Moyen Âge, le héros qui pour une raison ou une autre est obligé de se désocialiser s'exile dans la forêt, se dénude et perd la raison - ce qui dessine en creux les traits positivement humains : la sociabilité, le port du vêtement et la rationalité (Bartholeyns 2009 : 114-15). Se vêtir est alors compris comme le propre de l'homme : se dénuder, c'est courir le risque de l'animalité.

Positif ou négatif, le vêtement dans l'Occident chrétien est en tout cas le signe de l'humanité : une humanité qui cherche à retrouver son innocence perdue ou qui tente de se démarquer de l'animalité qui la menace. Dans cette ambivalence, l'être de l'homme, entre nature et culture, est flottant, incertain; il lui faut le secours de son apparence pour confirmer son appartenance à l'humanité. D'où par exemple la condamnation très ferme par les théologiens des travestissements animaliers, comme chez Césaire d'Arles (mort en 542) :

Quel homme sensé en effet pourrait croire que les hommes raisonnables veuillent se changer en bêtes sauvages? Certains se revêtent de la peau d'un animal, d'autres en prennent la tête et se réjouissent de ressembler à la bête brune. Ne montrent-ils pas par là qu'ils ont les sentiments de la bête plus encore que les dehors, et le cœur plus encore que la forme? (Sermo 192, De Kalendis ianuariis, 2, cité par Bartholeyns 2009 : 121)

Césaire part du principe simple (et combien important dans la culture européenne) que l'extérieur exprime l'intérieur, que l'apparence est déterminée par l'essence. Dès lors on comprend que l'homme qui s'assume en tant qu'homme ne doit pas afficher les dehors de 
l'animal, mais bien des traits extérieurs spécifiquement humains. Il est facile également, dans cette perspective, d'admettre l'idée que l'homme aurait un rapport spécifique à la parure : il serait la seule créature à modifier son apparence corporelle par l'ajout d'artefacts. L'idée paraît tellement évidente que, plusieurs siècles plus tard, et en dépit de transformations culturelles fondamentales, elle sert encore de base à un certain discours anthropologique universaliste qui, à partir de la fin du $\mathrm{XIX}^{\mathrm{e}}$ siècle (Semper en est un exemple), cherche à contrecarrer le discours racialiste courant en affirmant la communauté culturelle de tous les humains sur la surface de la terre, « sauvages » comme $"$ civilisés $»^{1}$. Seuls exclus de cette communauté : les animaux. La parure sert de nouveau de limite entre l'homme et l'animal.

Ce discours, on le retrouve au $\mathrm{XX}^{\mathrm{e}}$ siècle chez les chercheurs en sciences humaines, en particulier chez ceux qui arborent ce même universalisme du point de vue : les anthropologues et les psychanalystes. Ils ignorent (ou ne veulent pas voir) que leurs hypothèses sont largement déterminées par cette histoire chrétienne des apparences dont ils héritent à leur insu.

Emblématique des premiers, Claude Lévi-Strauss n'a cessé de vouloir repérer dans les relations et les mythes des peuples qu'il étudiait leur " humanité », c'est-à-dire leur savoir, leur rationalité, leur culture, à une époque où certes il était bon de rappeler que les « primitifs » colonisés n'étaient pas moins hommes que les colonisateurs. Cette humanité s'exprime notamment par la parure. Dans des pages célèbres de Tristes tropiques, LéviStrauss examine la fonction essentielle des peintures corporelles des Indiens caduveos du Brésil : les nobles «s'épilaient complètement le visage y compris les sourcils et les cils, et traitaient avec dégoût de "frères d'autruche" les Européens aux yeux embroussaillés ». Car « il fallait être peint pour être un homme ; celui qui restait à l'état de nature ne se distinguait pas de la brute » (Lévi-Strauss 1955 : 213-14).

Les Européens aux sourcils d'autruche... On devine la jubilation ironique de l'anthropologue. Les prétendus «sauvages » nous renvoient l'image qu'on pourrait avoir d'eux, à savoir : être plus près des animaux, plus éloignés de l'humanité. Le reproche est le même, quoique ne portant pas sur le même objet. La parure et toute modification artificielle des apparences visent dans tous les cas à passer de la nature à la culture, ou, pour utiliser d'autres termes, à pénétrer dans l'univers symbolique.

Dans cet univers, si l'on suit les conclusions à forte tendance psychanalytique de l'éthologue Jean-Marie Vidal, seul l'homme peut s'y mouvoir. C'est même ce qui le distingue des animaux. Dans un texte publié dans le numéro de Communications consacré à la parure, Vidal s'emploie à souligner les échecs de l'éthologie humaine, qui cherche à rapprocher le comportement humain du comportement animal. Il attribue comme cause première la différence irréductible entre les hommes et " nos frères inférieurs », à savoir chez eux l'absence du « registre symbolique » : pas de langage articulé, pas de névrose, pas de « stade du miroir », enfin pas de pudeur ou de parure animales (Vidal $1987: 9$ ).

Seul l'homme se construit une apparence artificielle parce qu'il est capable de se présenter à autrui comme un autre de lui-même, c'est-à-dire qu'il est le seul à posséder le « sentiment

1. Par exemple Edward Tylor peut affirmer : «il apparaît à la fois possible et désirable d'éliminer les considérations relatives aux variétés héréditaires des races humaines, et de traiter l'humanité comme homogène par nature, bien que pourvue de différents degrés de civilisation. Les détails de l'enquête prouveront, je pense, que les degrés de culture peuvent être comparés sans prendre en compte la différence de configuration corporelle et de couleur de peau et de cheveux des tribus qui utilisent la même installation, suivent la même coutume, ou croient au même mythe. » (Tylor 1920 : vol. $1: 7$, ma trad.). 
de soi ». Comme chez Lévi-Strauss, il s'agit de refuser une base « biologique » à l'ontologie humaine : l'homme est un être construit, un être de pur artifice.

Quand, dans ces textes, les apparences corporelles artificielles sont envisagées du point de vue matériel ou formel, les mêmes constatations se répètent : elles présentent une matérialité et des formes inorganiques ; elles privilégient les matières les moins « vivantes » possibles, voire les plus artificielles, et les formes les plus abstraites, voire géométriques, qui ne rappellent en rien le monde naturel (ce que montre la grande facilité avec laquelle on imite depuis une cinquantaine d'années la forme de vêtements en matériaux organiques avec des matériaux synthétiques). Déjà, dans le christianisme ancien, certains ascètes choisissaient pour se revêtir des tissus végétaux comme le coton plutôt que des fibres animales afin de marquer leur rejet de la nature animale qui pouvait les menacer (Bartholeyns 2008 (1) : 180). Même l'artifice humain n'apparaissait pas suffisant aux yeux de certains Pères de l'Église, à l'instar de Basile de Césarée :

L'homme ne devait donc avoir ni les vêtements de la nature (natura), ni ceux de l'art (arte) : mais d'autres (vêtements) lui étaient préparés s'il exprimait sa vertu, qui devaient briller en lui par la grâce divine, qui devaient l'embellir, comme le sont les anges, d'une parure (cultu) éclatante, laquelle effacerait la beauté des fleurs et la splendeur des astres. (Basile de Césarée, Homélie. Que dieu n'est pas l'auteur des $\left.\operatorname{maux} x^{2}\right)$

On trouve un écho lointain de cette idée par exemple chez Semper, pour qui, «dans l'art textile, l'ornement ne doit jamais être conçu selon une conception naturaliste ou par imitation fidèle de la nature » (Semper 2007 : 227). En effet, le textile, qui sert avant tout au revêtement, construit des surfaces ; or l'art naturaliste prétend à l'illusion du volume et ne convient donc pas pour remplir des surfaces. Il faut donc à l'art textile des motifs bidimensionnels non naturalistes. Par extension c'est toute parure (tout revêtement corporel) qui doit présenter ce caractère abstrait. L'idée trouve un écho chez Lévi-Strauss, toujours à propos des Caduveos : non pas que leur art textile présente des traits abstraits, mais plutôt que leur peinture corporelle présente un caractère textile, c'est-à-dire, en définitive, abstrait : « ces Indiens chevaliers ressemblaient à des figures de cartes. Ce trait ressortait déjà de leur costume : tuniques et manteaux de cuir élargissant la carrure et tombant en plis raides, décorés en noir et rouge de dessins que les anciens auteurs comparaient aux tapis de Turquie » (LéviStrauss 1955 : 203-204). Plus loin il décrit le système de dédoublement des peintures faciales en quatre quartiers « rarement partis ou coupés, plus souvent tranchés ou taillés, ou encore écartelés ou gironnés ». Ces règles « évoquent irrésistiblement les principes du blason »c'est-à-dire un langage formel très peu naturaliste, non organique, quasiment abstrait (LéviStrauss $1955: 217-18$ ).

Il revient à Georg Simmel d'avoir donné dans son célèbre essai de 1908 l'interprétation la plus complète de cet aspect inorganique de la parure (Simmel 1998 : 79-88). Le sociologue distingue trois formes de parure, de la plus personnelle à la plus impersonnelle : le tatouage (des « primitifs »), le vêtement et les bijoux (des Occidentaux). Le tatouage est personnel car il est attaché au corps : techniquement, il est organique, il ne peut se passer d'un corps, d'une 
peau³. Les bijoux, à l'autre extrémité, sont totalement impersonnels car fabriqués à partir de matériaux inertes (pierres précieuses, métaux) : ils sont portés mais n'appartiennent pas au corps, on peut les détacher, les donner, etc. Entre les deux, le vêtement, quand il est neuf, entoure le corps d'une enveloppe d'impersonnalité, et quand il est usé, il se personnalise, colle « trop » à son possesseur. « Trop », car aux yeux de Simmel il s'agit d'un défaut : le vêtement usé, comme le tatouage, renferme son porteur sur son individualité ; au contraire, le vêtement neuf et les bijoux l'environnent d'une sphère impersonnelle dans laquelle les autres (qui peuvent à l'occasion posséder de semblables accessoires) peuvent être incorporés. Si la parure inorganique est essentielle à l'homme moderne, c'est pour la réinscrire dans la collectivité et aller contre la tendance néfaste à l'individualisme de la société urbaine et industrielle. Vêtement et parure constituent ainsi, dans notre société, des outils essentiels pour fabriquer des «personnes disséminées », pour reprendre l'expression d'Alfred Gell (Gell 2009 : 119-187).

Les conclusions de ces interprétations se déduisent facilement. La parure fabrique l'humain et marque son accession au domaine de la culture, son entrée dans l'ordre symbolique ; elle arrache l'homme à son appartenance au règne animal. Comment? En l'enveloppant d'un sur-corps artificiel, inorganique, aux formes non naturelles ${ }^{4}$.

Mais pour être considérée comme un objet pleinement culturel, il faut aussi que la parure soit un langage. Elle ne doit pas seulement montrer l'humanité de son porteur, elle doit encore préciser la condition particulière de celui-ci au sein de l'humanité. Lévi-Strauss parle nettement de cette double fonction de la parure : «Les peintures de visage confèrent d'abord à l'individu sa dignité d'être humain ; elles opèrent le passage de la nature à la culture, de l'animal "stupide" à l'homme civilisé. Ensuite, différentes quant au style et à la composition selon les castes, elles expriment dans une société complexe la hiérarchie des statuts. Elles possèdent ainsi une fonction sociologique » (Lévi-Strauss 1955 : 220). En effet la société caduveo était très hiérarchisée : elle était décrite au $\mathrm{XVI}^{\mathrm{e}}$ siècle comme régulée féodalement par opposition entre seigneurs et serfs. Société de trois castes, divisées en souscatégories ternaires : nobles, guerriers, plèbes. « Les nobles faisaient étalage de leur rang par des peintures corporelles au pochoir ou des tatouages, qui étaient l'équivalent d'un blason » (Lévi-Strauss 1955 : 204).

Si le blason revient souvent dans le texte de Lévi-Strauss, ce n'est pas seulement du fait des ressemblances formelles avec les « figures de cartes ». C'est que le blason est un langage complexe qui communique visuellement des informations identitaires. Parmi beaucoup d'autres, mais de façon exemplaire, Michel Pastoureau a étudié les façons dont le Moyen

3. Le lecteur trouvera sans doute étonnant que Simmel réduise le tatouage à un marqueur individuel et en dénie la fonction sociale. S'il avait connu les recherches entreprises par Karl von den Steinen (von den Steinen 2005) à peu près au même moment sur les tatouages marquisiens, il aurait bien dû admettre que le tatouage des «primitifs » vise autant à individualiser leur porteur qu'à les intégrer dans une communauté, notamment par l'usage de signes mythologiques communs ou par leur style. Cette erreur de Simmel est le fait de son ignorance de tout critère autre que matériel et technique, qui est, dans cet essai, le seul signifiant à ses yeux.

4. On a noté plus haut que pour Simmel, le premier stade de la parure est le tatouage, qui relève de l'organique parce qu'il est attaché au corps. Mais, dans la logique du sociologue allemand, cette organicité du tatouage est le signe de son caractère peu évolué (il ne va pas jusqu'à dire animal). La véritable parure, pour Simmel, doit être détachée du corps. 
Âge avait codifié les apparences, c'est-à-dire les avaient transformé en langage. L'héraldique en est une et non des moindres (Pastoureau 1986 : 89-155). Apparu au XII ${ }^{\mathrm{e}}$ siècle, ce type d'image très stylisée marque à la fois, pour celui qui l'exhibe, l'appartenance à une catégorie sociale (la noblesse adoubée : l'armoirie, comme son nom l'indique, figure d'abord sur les armes des chevaliers), à une famille (par la singularité du motif) (Pastoureau $1986: 89-98$ ), et à la fois une façon de construire un langage visuel quasiment abstrait, où les référents naturels comme les animaux n'interviennent que comme des signes symboliques élémentaires (Pastoureau 1986 : 102-105; et sur les animaux : 107).

Apparaissent plus tard des accessoires que Pastoureau appellent «para-héraldiques » comme le cimier : construction de papier mâché ou de bois, parfois assez haute, plantée sur le heaume de l'armure, le cimier prend le plus souvent l'aspect d'un animal, qui n'appartient pas toujours au vocabulaire du blason et qui, de ce fait, est capable d'indiquer l'individualité de celui qui le porte (Pastoureau 1986 : 128). Cette signature ornementale, pourrait-on dire, de l'individu noble, fait partie d'une stratégie de la distinction par l'apparence qui, à la fin du Moyen Âge, s'étend à ses vêtements et à ses parures (les emblèmes et devises se répandent partout, sur les manches des vêtements, sur les ceintures, les objets et les murs du château (Pastoureau 1986 : 131-32). Plus globalement, c'est toute la hiérarchie sociale qui doit se manifester par les apparences vestimentaires, comme le formule très clairement le franciscain Bernardin de Sienne, au $\mathrm{XV}^{\mathrm{e}}$ siècle : " parce que les hommes connaissent et gouvernent les choses intérieures par les extérieures, il a été nécessaire en outre que cette diversité d'œuvres, de dignités et d'offices fût distinguée par quelques signes extérieurs, entre lesquels les vêtements tiennent le premier rang. » (Bernardin de Sienne, Enseignements et apologues, Paris, 1923, ch. 22, cité par Crouzet-Pavan 2007 : 407).

En somme, malgré tout ce qui les sépare, les Caduveo partagent avec les médiévaux le souci des apparences distinctives. Ce comparatisme peut aller plus loin. Ainsi, LéviStrauss (du moins dans les textes mobilisés ici) comme Pastoureau, associent parure et «totémisme $»^{5}$, à travers l'étude des masques pour le premier, des cimiers pour le second. Dans l'essai que l'anthropologue consacre aux représentations dédoublées, il en vient à parler du dédoublement comme une structure ontologique entre l'acteur et son rôle. Et il prend l'exemple des «masques à volets » des Eskimo et des Indiens Kwakiutl du Canada, qui, ouverts ou fermés, « présentent alternativement plusieurs aspects de l'ancêtre totémique, tantôt pacifique, tantôt irrité, tantôt humain et tantôt animal [...]. Leur rôle est d'offrir une série de formes intermédiaires, qui assurent le passage du symbole à la signification, du magique au normal, du surnaturel au social. » (Lévi-Strauss 1985 : 313-14) Quant au cimier, analysé par Pastoureau, "il est beaucoup plus qu'un simple signe de reconnaissance. Il est l'image d'une seconde nature, qui participe non seulement de la fête, du jeu et de la guerre, mais aussi de la mort et de l'au-delà. Par-là même, il met celui qui le porte en relation avec ses ancêtres, véritables ou inventés, et avec l'ensemble de sa parenté. De masque il devient "totem" " (Pastoureau 1986: 145)6. Le totémisme, dans ces interprétations d'un

5. Bien sûr, quand plus tard Lévi-Strauss se penchera en détail sur le totémisme, notamment dans Le Totémisme aujourd'hui (1962), ce sera pour le critiquer, concept trop flou, utilisé à tout va-l'essai de Pastoureau en est un exemple tardif.

6. Déjà en 1919 Johan Huizinga (1967 : 244) avait établi la comparaison : « La figure héraldique prend dans son esprit [celui de «l'homme du Moyen Âge »] une valeur analogue à celle d'un totem. » 
anthropologue et d'un historien, joue donc un rôle à peu près similaire : celui de nommer la relation entre une image et une identité, celui de faire basculer son porteur dans la culture. Le totémisme serait l'utilisation symbolique des apparences.

Tout est lié : le caractère abstrait de la décoration corporelle, sa symbolique totémique, l'humanisation produite par la parure, son caractère social.

\section{La parure organique, ou la fabrique de la nature}

Tout va donc se délier à présent. Reprenons par ses fondements l'argumentation précédente, résumée par l'affirmation de Semper : « seul l'homme se pare ». À cela il sera donné deux types de contradictions. La première sera une réponse ethnologique : «seul l'homme se pare » est une affirmation seulement valable dans notre ontologie naturaliste. La seconde sera une réponse éthologique : même dans ce système, nous pouvons penser une parure animale. Ces deux réponses ne sont pas si opposées qu'elles en ont l'air : elles ont en commun de déplacer la frontière entre la nature et la culture, de ne plus la faire correspondre à la frontière entre l'homme et l'animal. Dans un cas comme dans l'autre, l'homme n'a plus le privilège de la parure, parce que l'homme n'a plus celui de la culture.

En évoquant l'ontologie naturaliste, je fais référence évidemment aux travaux de Philippe Descola qui ont permis de donner raison, d'un point de vue ethnologique, aux critiques philosophiques formulées depuis au moins Nietzsche à l'égard du grand partage entre Nature et Culture (Rosset 2004). L' " anthropologie de la nature » à laquelle s'attache Descola vise à comprendre les différents statuts et rôles attribués aux êtres non-humains dans l'environnement des sociétés humaines; la figure cartésienne de l'homme « maître et possesseur » de la nature est bien occidentale et d'autres attitudes, plus équilibrées, plus en phase avec les préoccupations écologiques actuelles, sont adoptées par les sociétés étudiées par les ethnologues. L'attitude naturaliste - la nôtre, donc - est caractérisée par Descola, qui en repère les traces depuis Aristote et la voit véritablement s'édifier dans la pensée chrétienne, par la certitude que tous les êtres vivants sont reliés physiquement par un continuum physiologique (ce que la théorie de l'évolution démontre) et par la conviction que l'homme se distingue radicalement des autres êtres vivants au niveau psychologique, intellectuel (l'homme, animal raisonnable, doué pour le langage, la réflexion, le symbolique, la parure, l'art - nous sommes en terrain connu) (Descola 2005 : 241-79). Bref, seul l'être humain est une « personne ».

À l'inverse, l'attitude animiste - celle de certaines populations indiennes d'Amazonie notamment, mais aussi d'Indiens d'Amérique du Nord, d'Eskimos, de peuples d'Asie et du Pacifique - se présente comme la certitude qu'il existe une continuité intérieure entre les êtres vivants (ils sont tous «psychologiquement» semblables aux humains, ce sont tous des «personnes ») et une discontinuité extérieure (ils ne prennent pas tous l'apparence d'êtres humains) (Descola 2005 : 183-202). Cette ontologie animiste va retenir notre attention, car elle permet de comprendre qu'un « animal », un guépard ou un ours, un colibri ou un tapir, est en réalité un humain qui a pris l'apparence de cet animal ; une apparence qui n'est pas stable, attachée à son essence, mais qui est au contraire relationnelle : entre eux, les ours se perçoivent tous comme des humains, de même que les guépards ou les oiseaux. " $\mathrm{Ce}$ sont donc des personnes, revêtues d'un corps animal ou végétal dont elles se dépouillent à l'occasion pour mener une vie analogue à celle des humains » (Descola 2005 : 187). C'est qu'alors ils entretiennent des rapports sociaux équilibrés. Ils prennent l'apparence du 
prédateur quand ils occupent un rapport agressif avec une autre créature, et au contraire, l'apparence d'une proie quand ils sont soumis à l'épreuve de la chasse ou de la mise à mort. Dans ce système, on peut donc considérer que tous les êtres vivants, à l'instar des hommes, revêtent une apparence artificielle qui signifie leur position sociale ; chaque animal porte un « habit», écrit A.-C. Taylor à propos des Jivaros (cité par Descola 2005 : 185), ou une « cape », comme disent les Chewong de Malaisie (ibid. : 186).

De même, on ne s'étonnera pas non plus que les hommes puissent eux-mêmes s'habiller en animaux, à l'occasion - quand leur rôle dans la société le demande (voir les contributions de Bartholeyns et Prévost dans ce volume). Tel est le cas du chasseur amazonien dont le « devenir jaguar » exige non seulement un comportement agressif de jaguar, mais également une apparence de jaguar (Taylor 2010 : 42). D'où l'utilisation, dans la parure, de dents de ces prédateurs, de couleurs qui leur ressemblent etc. (Taylor 2010 : 50). Dans certaines cérémonies chamaniques, il est fait usage de masques qui visent à donner au chamane l'apparence d'un esprit animal (Descola $2010: 23,25,38$ ) ; non pas pour qu'il perde son humanité, mais pour qu'il entre en communication avec les esprits, c'est-à-dire pour qu'il occupe vis-à-vis des autres hommes la position (dominante) d'un esprit.

On retrouve ici, dans les analyses de Descola, l'exemple des masques eskimos à volets, ou provenant de la côte nord-ouest de l'Amérique (Indiens Kwakiutl) dont parlait Lévi-Strauss (Descola 2010 : 26). À la différence de ce dernier, qui, rappelons-le, pensait ces masques en relation avec un animal totem, Descola (pour qui le totémisme implique une ontologie radicalement différente de l'animisme) voit dans le double aspect que ces masques peuvent prendre (fermés, ils sont zoomorphes; ouverts, anthropomorphes) une illustration quasi littérale de l'ontologie animiste. Dans la société animiste, se fabriquer un corps d'animal et revêtir un masque zoomorphe n'a pas le même sens, car « si mettre un costume, c'est accéder à la physicalité de l'animal, mettre un masque, c'est accéder à son intériorité et la contrôler en partie » (Descola $2010: 36$ ).

En fait, tout se passe comme si, dans l'animisme, le corps nu de l'homme n'était que son âme et que, pour avoir un corps, il fallait le construire, en l'empruntant aux autres animaux. Renversant complètement les positions de Lévi-Strauss, Descola affirme :

Ce travail sur la forme des corps a d'autant moins pour finalité de démarquer l'humain de l'animal en imposant sur la "nature" le sceau de la "culture" que ce sont précisément des greffes animales qui servent à cet effet. Le port de plumes, de dents, de peaux, de masques avec des becs, des crocs ou des touffes de poils permet en fait de différencier, grâce aux attributs mêmes qui signalent la discontinuité des espèces, non pas l'homme de l'animal, mais diverses sortes d'espèces humaines trop semblables par leur physicalité originelle: en arborant des ornements caractéristiques, les membres de tribus voisines peuvent ainsi exhiber des écarts d'apparence analogues à ceux qui distinguent entre elles les personnes non humaines. (Descola 2005 : 189)

Par ces écarts visuels, les hommes et les animaux peuvent se positionner dans la société qu'ils partagent. Et de nouveau sous la plume de Descola apparaît l'image du blason : les animaux entre eux voient les motifs de leur pelage « comme des dessins géométriques plus ou moins complexes ayant une fonction héraldique », de même que les humains se peignent le corps non pour imiter seulement l'apparence naturelle des animaux, mais le codage 
héraldique dont ils pensent que ceux-ci se recouvrent intentionnellement (Descola 2010 : $36)^{7}$.

Cette approche héraldique de la parure des animaux trouve un écho plus récent dans les travaux du zoologue suisse Adolf Portmann autour de 1950. Celui-ci se passionne pour la diversité des formes animales. Pour lui l'apparence des animaux a un sens indépendant des autres fonctions du corps : elle constitue un ensemble d' " organes visuels » (Portmann 1961 : 109-121). Certes il arrive que la théorie évolutionniste permette de donner une raison à certaines configurations formelles remarquables : pour sauvegarder et faire fructifier l'espèce, certains animaux se servent de leur apparence pour attirer les reproducteurs ou repousser les prédateurs (par le camouflage ou au contraire des signaux colorés violents et des formes menaçantes). Mais de telles raisons, on ne peut les attribuer dans la plupart des cas observés : la variété de la nature est trop grande, elle excède toute fonctionnalité de ce genre (Portmann 1961 : 202). Si la conservation de l'espèce n'est donc pas la cause finale (au sens d'Aristote) de la diversité des formes animales, il faut lui en assigner une autre. Portmann propose de considérer les morphologies naturelles comme des façons de caractériser l'espèce, mais aussi l'âge, le sexe, bref d'individualiser les animaux (Portmann 1961 : 160 sq.) : «Plus les différences sont externes, plus elles sont caractéristiques ; plus elles sont superficielles, plus elles sont spécifiques. » (Portmann 1961 : 208) En un mot, on l'aura deviné, ce sont des armoiries (Portmann 1961 : 114). Portmann propose une « sémiotique des apparences » animales (Lestel $2009: 271$ ).

À cette première détermination générale de l'apparence animale, Portmann en ajoute une seconde : l'apparence a une puissance expressive, elle permet d'exprimer l'intériorité de l'animal (Portmann 1961 : 162-193). Chez les espèces inférieures, dont le cerveau est peu développé, cette intériorité est essentiellement physique : une méduse ne cache rien de sa structure interne parfaitement symétrique. Chez les espèces supérieures, l'intériorité est plus psychologique : elle est constituée par les réactions nerveuses, les émotions, les réflexions que les animaux les plus évolués peuvent éprouver. Par leurs attitudes corporelles, la couleur de leur enveloppe, leurs cris ou leurs odeurs, ils sont capables d'exprimer cette intériorité immatérielle, comme l'homme qui rougit ou pâlit, ou laisse échapper quelque expression involontaire. La morphologie des espèces les plus évoluées présente d'ailleurs, selon Portmann, un trait commun : leur apparence souligne le rôle directeur de la tête et leur parure met en valeur cette partie du corps, comme pour bien souligner que c'est là que se manifeste leur supériorité (Portmann 1961 : 205).

Comme l'armoirie médiévale, comme la peinture corporelle caduveo, la parure animale a donc une double raison : elle manifeste l'appartenance à un groupe social et spécifique,

7. On pourrait parler, dans la culture médiévale occidentale, d'un animisme marginal. Le Moyen Âge européen pouvait penser des blasons naturels, inscrits sur le corps vivant. J'en veux pour preuve cette analyse par Jean-Marc Pastré du Parzival de Wolfram von Eschenbach qui présente un personnage remarquable à ce titre (Pastré 1994 : 74-75). Le héros Gahmuret, de la maison d’Anjou, épouse Belacâne, reine de Zazamanc en Afrique, noire de peau. Leur enfant naît noir et blanc alterné, comme une pie : il s'appelle Feirefîz (d'après l'ancien français vair, varié). Feirefîz porte sur sa peau son armoirie : le vair est d'abord la fourrure blanche et grise du petit-gris, puis un terme d'héraldique, qui désigne la fourrure du blason en petits points blancs et bleus alternés. Le fils reprend corporellement les couleurs de son père : fond blanc semé de queues noires. Son métissage est valorisé par l'héraldique. Quand on sait que plus tard, Gahmuret revenu en Anjou reprendra les armoiries de sa maison, le léopard, blanc avec des taches noires, on comprend que la boucle est bouclée : la correspondance est totale entre la robe de l'animal, la peau du fils et l'habit du père. 
et elle classe l'individu dans une hiérarchie - celle de sa société, ou celle des êtres vivants. Peut-on encore parler ici d'animisme ? Sans doute pas au sens littéral, puisque la recherche de Portmann s'inscrit nettement dans le cadre de pensée de la science naturaliste. Mais la possibilité de la comparaison montre plutôt qu'au sein même du naturalisme se formulent des remises en cause de la correspondance entre nature et animalité, culture et humanité.

Avec Portmann nous parvenons peut-être au bout d'une certaine hypothèse sémiotique. Au bout car il est difficile d'aller plus loin : n'importe quelle apparence animale (et, à la limite, n'importe quel être vivant - Portmann n'exclut pas de son champ problématique l'immense variété des formes végétales) peut à présent être assimilée à la parure humaine, du moment qu'elle entre dans un système de signification, qui repose sur la dialectique de l'intérieur et de l'extérieur et sur la dynamique de l'expressivité. Mais au bout, car on voit aussi les limites d'une telle approche.

Outre que certaines expressions du savant suisse pourraient aujourd'hui le faire passer pour un partisan de l'Intelligent Design - il parle de " plan concerté », de création qui ne doit rien au hasard... (Portmann 1961 : 107, 159) -, c'est surtout le classement des espèces vivantes, qui détermine sa sémiotique des apparences animales, qui peut faire l'objet des critiques les plus constructives. Tout le classement morphologique de Portmann repose sur l'idée qu'il existe des animaux plus évolués que d'autres, en se basant d'abord sur l'indice cérébral, c'est-à-dire le rapport entre la taille du corps et la taille du tronc cérébral (Portmann 1961 : 55-59). Ce rapport donne à l'homme une supériorité écrasante sur n'importe quelle autre espèce (170 contre 49 pour le chimpanzé). Mais à cette échelle qualitative Portmann fait correspondre des données paléontologiques et des observations morphologiques : les espèces primitives auraient un cortex peu développé, donc des formes peu différenciées, tandis que les espèces les plus récentes ou les plus évoluées, auraient un cortex cérébral plus développé et des formes plus différenciées. Pourquoi ? Parce que les formes sont des phénomènes visibles et que le développement de la vue est un indice de supériorité chez les espèces qui en sont pourvues : les espèces inférieures en seraient dépourvues et se contenteraient de l'odorat (Portmann $1961:$ 72).

Or, non seulement il s'agit d'une position assez arbitraire d'un point de vue éthologique (on ne comprend pas en quoi la mouche avec ses yeux surdimensionnés à facettes est supérieure à l'homme ou au cachalot, qui voit très mal et pourtant possède une vie sociale très complexe), mais on voit très bien en quoi elle est héritée de l'histoire culturelle européenne, où la vue, depuis des siècles, a été considérée comme le sens le plus important. Tout ceci mène à l'idée, là encore très ancienne, que l'homme est l'aboutissement de l'évolution (pour ne pas dire de la création). Idée pourtant battue en brèche depuis longtemps par beaucoup de biologistes, de naturalistes qui remettent en cause ce genre de correspondances : ainsi, le développement du système nerveux n'est plus aujourd'hui interprété comme entraînant un développement unilatéral de la vision au détriment de l'odorat. Ce n'est pas l'équipement sensoriel qui fait la différence mais la complexité du système nerveux : un œil d'escargot et un œil humain sont constitués de la même façon, mais l'escargot a du mal à isoler des formes parce que son système nerveux est beaucoup moins développé (Laget 2005, Huergueta 2005).

À partir de l'exemple de Portmann, nous pouvons peut-être établir une critique plus générale de la sémiotique des apparences humaines et animales. Réduisant les apparences à un langage codé, la comparaison avec le blason maintient encore la parure du côté de l'abstrait, du culturel, du mental. Elle reflète cette habitude occidentale d'assimiler toute parure à un 
vêtement aux formes et aux motifs inorganiques. Elle témoigne de ce primat, typique des sciences humaines pendant longtemps, du linguistique sur le visuel. Elle empêche de penser la parure dans sa relation avec les problèmes du vivant et du beau.

Une première manière, la plus évidente, d'associer ces deux notions à la parure est de l'envisager sous l'angle de la séduction et de l'attrait. C'est la fonction première que des sociologues de la parure lui attribuent, comme Simmel ou Flügel, en amont de toute sémiotique (Simmel 1998 : 79-80 ; Flügel 1982 : 14, 20). La parure met en valeur son porteur, elle attire le regard. Si elle est coûteuse, elle signifie phénoménologiquement : « celui qui me porte le mérite ». Sa fonction est sociale : attirer le regard pour plaire, plaire pour tirer des avantages d'autrui. Par exemple, dans ses écrits sur les Caduveo, Lévi-Strauss laisse échapper une ligne de fuite qui prend en compte cette dimension esthétique de la peinture corporelle mais ne s'intègre pas à son interprétation sémiotique de la parure. Il remarque que les jeunes femmes dégagent un air de séduction indéniable. Les blancs installés dans ces contrées n'y sont pas insensibles. « Les peintures faciales et corporelles expliquent peut-être cet attrait, explique l'anthropologue ; en tout cas, elles le renforcent et le symbolisent. Ces contours délicats et subtils, aussi sensibles que les lignes du visage et qui tantôt les soulignent et tantôt les trahissent, donnent à la femme quelque chose de délicieusement provocant. » Et il rajoute : « Jamais, sans doute, l'effet érotique des fards n'a été aussi systématiquement et consciemment exploité » (Lévi-Strauss 1955: 214-15).

On entre ici dans un domaine qui n'est plus celui de la sémiotique, mais de la fonction esthétique des apparences corporelles. Mais cette esthétique est aussi, et avant tout, naturelle : la séduction de la parure s'exerce entre hommes et femmes, entre mâles et femelles, si bien qu'elle contribue à la reproduction et au développement de l'espèce, humaine ou pas. Comme le remarque André Leroi-Gourhan, « l'esthétique du vêtement et de la parure, malgré son caractère entièrement artificiel, est un des traits biologiques de l'espèce humaine les plus profondément liés au monde zoologique » (Leroi-Gourhan 1965 : 189) ${ }^{8}$. Cette approche éthologique de la parure permet donc de franchir la frontière entre l'homme et l'animal : dans cette perspective, c'est parce que l'homme est un animal qu'il se pare. Ce que l'homme construit par son artifice, l'évolution le produit pour les animaux. Le biologiste Claude Gudin l'affirme de belle manière :

La séduction est biologique, avec ses émetteurs, ses récepteurs, et évolue avec la vie. Elle est le produit de notre histoire naturelle et de l'histoire culturelle de l'homme. Quand nous séduisons, comment distinguer notre animalité de notre culture? [...] Après tout, pourquoi les opposer l'une à l'autre? Si la culture est l'art d'inventer, de créer des stratégies et de les transmettre à notre descendance, ne commence-t-elle pas avec les insectes, les poissons et tous les animaux? [...] Quelle différence entre le flamant qui déclare sa flamme en rose à la femelle et l'amoureux transi qui offre un bouquet de fleurs (une gerbe de sexes en rut) à la dame qu'il convoite? Quelle différence entre le sac en crocodile qu'on offre en cadeau et la mouche enveloppée dans de la soie verte offerte à l'araignée par Monsieur? Quelle différence entre la danse de l'aigrette pour séduire la femelle et celle de la dame des Folies-Bergère avec ses plumes d'aigrette pour séduire les banquiers? (Gudin 2003 : 110-111)

8. Il faut noter cependant que Leroi-Gourhan réduit l'esthétique des apparences corporelles à une question d'identification sociale. 
Bien sûr, les différences abondent, dira-t-on, ne serait-ce qu'en raison des configurations sociales chaque fois spécifiques que ces exemples convoquent implicitement. Mais si Gudin force le trait de manière quelque peu provocante, c'est pour bien faire sentir que la séduction n'est pas le propre de l'espèce humaine, mais que c'est là plutôt quelque chose que l'homme a en commun avec toute une série d'espèces. En d'autres termes, le sentiment esthétique, tel qu'il se manifeste à travers la séduction, est loin d'être le propre de l'homme.

Une autre différence, c'est l'outil, répondra-t-on: les plumes d'aigrette ne font pas partie du corps de la danseuse. Mais les éthologues travaillent depuis longtemps sur des cas (comme celui cité de l'araignée) où des «outils» sont utilisés à des fins de séduction par certains animaux. Soit l'exemple fameux des chlamyderas ou jardiniers, cité à la fois par Gudin (Gudin 2003 : 100) et par Vidal (Vidal 1986 : 10-11), évidemment pas du tout pour en tirer les mêmes conclusions. Un éthologue anglais, Joah Madden, a également travaillé sur ces oiseaux de Nouvelle-Guinée et publié depuis ces dernières années plusieurs articles qui vont dans le sens de Gudin. Ces oiseaux sont connus pour aménager des sortes de berceaux ouverts, tressés avec grande ingéniosité, dans lequel le mâle fait sa parade nuptiale en tenant dans son bec une fleur ou un coquillage, pour attirer la femelle (fig. 1). Mais Vidal se trompe en affirmant que les objets apportés ou tenus par le mâle ne se rapportent qu'à des fins alimentaires ou de nidification. Madden a observé que ces oiseaux décoraient les berceaux destinés à recevoir la femelle d'objets hétéroclites qu'ils vont parfois chercher très loin et dont, par défaut, on ne peut reconnaître qu'une fonction esthétique (baies sauvages, os, coquillages, brins de feuilles, morceaux de plastique, de verre... plus d'une soixantaine en tout). De plus, il a remarqué de grandes différences locales de style de décoration selon l'emplacement géographique des oiseaux. Écartant la thèse de la variation génétique après une enquête statistique très élaborée, il en vient à parler de véritable comportement culturel : une habitude locale est prise et transmise de génération en génération (Madden 2004 : 763 64 ; Lestel 2009 : 71).

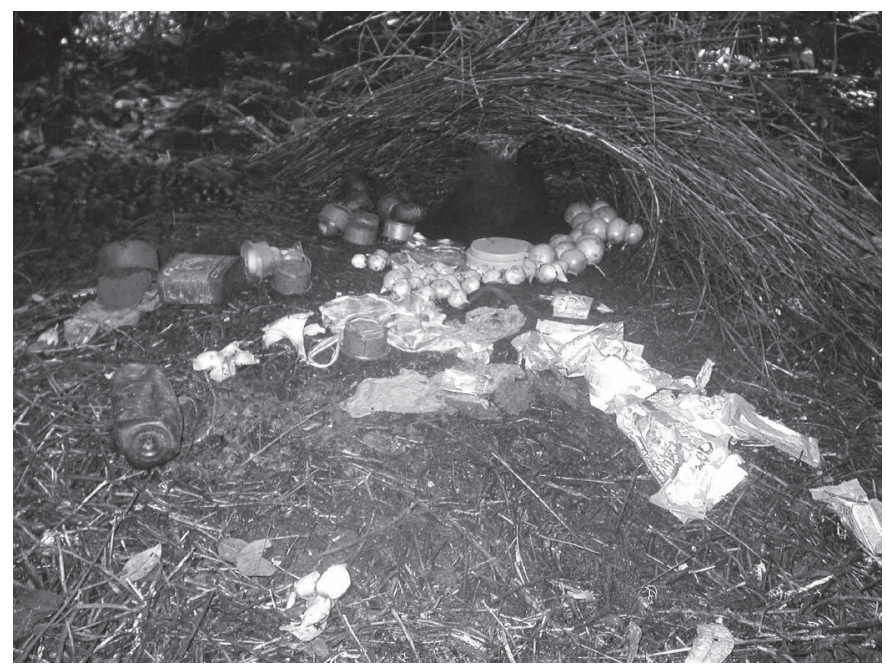

Figure 1

Nid de grand jardinier, Papouasie occidentale, Arfak Mountains, 13 août 2008.

(C) Photo Collaert Brothers. 
Pour Gudin comme pour Madden, les animaux se servent de la décoration corporelle ou artefactuelle pour séduire. Tandis que l'un montre que l'homme est un animal, le second montre que certains animaux ont une culture : dans les deux cas, ce sont les usages de la parure qui appuient la démonstration. Mais il faut voir au-delà de cette fonction pourtant cruciale pour l'espèce et pour l'individu, car Portmann a sans doute raison de ne pas restreindre l'esthétique animale à un outil de séduction destiné à faciliter la reproduction (et donc à assurer la survie de l'espèce) $)^{9}$. Elle est pensée par le zoologue comme une sorte de "fierté » de se montrer chez l'animal dans un acte d' « autoprésentation », dit son meilleur connaisseur français, Jacques Dewitte. Celui-ci n'hésite pas d'ailleurs à assimiler l'autoprésentation animale avec l'exhibitionnisme de l'homme baroque qui se « pavane » (Dewitte 1999: 29). À la même époque, Jacob von Uexküll comparait la coévolution des animaux qui semblent vivre l'un pour l'autre (comme la chauve-souris et le papillon de nuit, l'abeille et la fleur) à la composition d'une vaste partition ou d'un grand tableau, dont on aurait perdu les traces de l'auteur (von Uexküll 1984 [1956] : 146-52, 168-69). Mais si l'on préfère éviter ce genre d'anthropomorphisme, il sera sans doute plus utile de recourir aux travaux de la morphogenèse. Cette science permet de prendre en compte la dimension esthétique et vitaliste de la parure mais sans lui associer de fonction. Ce que Portmann assimilait peu ou prou à une intention esthétique - la composition des parures - est interprété par la morphogenèse comme le résultat de forces physiques dont les effets trouvent des équivalents dans le domaine des arts et qui peuvent donc être ressentis comme des principes esthétiques (Petitot 2004 : 13-139).

La morphogenèse connaît un essor véritable depuis les cinquante dernières années. Rappelons qu'il s'agit d'une science qui «entend prendre en compte l'aspect temporel, dynamique de la naissance des formes » (Fleury 2003 : 8). Parmi tous les phénomènes qui expliquent cette dynamique (mécaniques, chimiques, génétiques...), la morphogenèse met l'accent sur les phénomènes mécaniques qui contribuent à la naissance des formes (dont et essentiellement le hasard, cause des irrégularités créatrices de formes). Son champ d'observation inclut toutes les formes de la nature, celles du monde biologique comme celles du monde inanimé, puisque sur toutes, les forces physiques exercent une action.

La morphogenèse prétend ainsi expliquer pourquoi les formes de la nature sont à la fois régulières et variées : elles sont régulières parce qu'en partie déterminées par des forces physiques universelles; elles sont variées parce que la morphogenèse au sens strict (des événements qui produisent un changement de forme) se déclenche, dans le domaine biologique, au niveau embryonnaire - des variations microscopiques produisent des effets visibles gigantesques (Fleury 2003 : 108). Elle réfute donc toute hypothèse finaliste : la fonction d'un organe ne guide pas sa formation. Par exemple, les plis du cerveau ne se produisent pas pour permettre aux neurones éloignés de communiquer plus vite (Fleury 2003 : 65-67) ; ou encore, la forme fine et allongée d'un poisson n'est pas l'effet d'un besoin hydrodynamique (Fleury 2003 : 53). C'est la sélection naturelle qui fait corréler la forme et la fonction (les mieux adaptés survivent). Comme le résume Fleury : «La sélection fait le tri dans les patrons, mais ce sont les possibilités de construction qui délimitent le champ des patrons possibles » (Fleury $2003: 53-54)$.

9. Sur la valorisation de l'apport de Portmann à une esthétique animale, voir Prévost 2009. Mais cet article stimulant manque quelque peu de recul critique vis-à-vis des thèses du savant suisse. 
La parure animale n'est donc pas apparue, dans toutes ses variations, pour répondre à des fonctions (séduction, défense, camouflage, etc.) mais par le résultat de l'effet de forces physiques sur les matériaux du vivant. Elle n'est pas guidée par une quelconque finalité ou sémiotique de l'être vivant (Fleury, comme d'ailleurs Portmann, s'oppose ici fortement au finalisme de von Uexküll) (von Uexküll 1984 [1956] : 152-55). Elle est le fruit du hasard, son développement est aveugle. Ainsi peut s'expliquer l'apparition de ces «formes sans destinataire » qui fascinent Portmann telles les organismes marins plongés dans l'obscurité des profondeurs océanes ou des organismes microscopiques à la beauté géométrique invisible. Ces formes sont aussi contingentes (et calculables) que les dunes de sable ou les facettes de cristaux.

Pour avancer plus loin, il faut ajouter que la morphogenèse distingue, à un certain niveau, le monde physique (inanimé) du monde vivant. Deux caractéristiques en effet les séparent. D'abord, dans le monde physique, les forces sont produites de l'extérieur des molécules affectées (ainsi la pesanteur), tandis que dans le monde biologique, en plus de ces forces extérieures il faut ajouter des forces intérieures (la reproduction des cellules). Tout se passe comme si, dans le monde du vivant, une " poussée interne » affectait le matériau vers l'extérieur, tandis que dans le monde physique cette poussée l'affecte vers l'intérieur (Fleury 2003 : 82, 154-57). Ensuite (et cela découle de la première raison), les molécules du vivant s'organisent sous la forme de fibres qui s'enroulent en anneaux, en boucles, en faisceaux, en couches superposées, créant des formes de liens souples, plastiques; ceci explique que les choses vivantes sont molles et contrairement aux choses inertes, ont tendance à ne pas casser (Fleury 2003 : 46-47) ${ }^{10}$. L'ensemble forme un «tissu », comme on le dit de la peau - tissu plus ou moins souple selon la nature des molécules (par exemple les fibres de bois sont plus rigides que celles de la peau). Ce tissu de fibres moléculaires est « anisotrope », c'est-à-dire que ses directions ne sont pas équivalentes : en effet, selon l'agencement des molécules fibrées se déterminent un nombre limité de positions possibles de développement, en fonction des « coutures » que le tissu génère. Ainsi, un doigt est recouvert d'un tube de peau encapuchonné ; les empreintes digitales, qui se resserrent au centre de la proéminence de la dernière phalange, constituent la « couture » de la peau à cet endroit ; la proéminence est le résultat de la couture, un peu, dit Fleury, l'équivalent du talon d'une chaussette (Fleury $2003: 25)$.

Globalement c'est un « différentiel de croissance » qui produit le pli ou la bifurcation, même si ses causes, à ce niveau moléculaire, peuvent être très diverses (Fleury $2003: 82$ ). Ce différentiel explique aussi l'alternance plus ou moins régulière des taches de la peau du léopard, des zébrures de la girafe, l'espacement aligné des poils, des cheveux et des plumes, les motifs sur les carapaces de tortue, etc. (Fleury 2003 : 138-40). Ainsi, peut-on conclure, plus un organisme croît, plus ses formes sont complexes, ramifiées, plissées, fractalisées, comme dans les ornementations artefactuelles les plus sophistiquées (Golsenne 2009). Les « motifs » qui recouvrent le pelage des mammifères, les ailes de papillon, les écailles de poisson, les coquilles de gastéropodes et ainsi de suite ne sont pas crées par des phénomènes indépendants de l'organisme porteur, comme un papier peint qui recouvrirait un mur nu, mais sont créés par la croissance même de cet organisme. Pour savoir pourquoi il y a des

10. Le vivant connaît aussi néanmoins de nombreux cas de formations cristallines, à l'instar des minéraux, tels les os, les coquilles ou l'émail des dents (Perl-Treves 1998 : 4-38). 
empreintes digitales sur les doigts, il faut savoir pourquoi il y a des doigts ; de même, pour expliquer la variation des motifs tachetés sur la queue d'un léopard, il faut se demander comment se fabrique une queue.

De même qu'une analyse morphogénétique de la nature ne distingue pas a priori le monde animé et le monde inanimé, de même, la morphogenèse ignore la distinction a priori de la nature et de la culture. Un exemple nous permettra ainsi de comparer la parure artificielle à la parure animale d'un point de vue morphogénétique.

La comparaison de l'agencement des molécules de matériau vivant à un « tissu » amène à considérer la réciproque : les vêtements en tissu, mais aussi les vanneries, ne fonctionnentils pas formellement comme les matériaux vivants? Vincent Fleury en plusieurs endroits de son livre affirme cette réciprocité. « En fait, écrit-il, la structure tissée du vivant est tellement proche d'un véritable tissu qu'on peut se demander si l'homme n'a pas simplement emprunté à la nature l'idée du tissage » (Fleury 2003 : 105). Il prend plusieurs exemples : des feuilles de palmier séchées qui ressemblent à des sacs en toile de jute (106); une natte de paille dont la disposition des fibres permet de se dérouler dans la longueur et pas dans la largeur (26); un chapeau de paille d'Afrique du sud dont voici la description précise : « On remarquera que la rigidité de la paille oblige à orienter les fibres sur la tête d'une façon qui engendre une couture sur le côté. Dans cette couture, une ligne de fibres termine en queue de poisson dans une ligne de fibres qui effectue un demi-tour. En prenant un peu de recul, on verra que ce chapeau est cousu, en fait, comme une empreinte digitale (schématiquement) » (28). On pourrait se demander, en lisant Fleury, si c'est l'analyse des structures formelles, donc visuelles, qui guide ici le langage (à savoir la comparaison entre le tissu artefactuel et le tissu organique) ou si c'est le langage (la métaphore du tissu organique) qui guide l'analyse. En fait, depuis Bichat qui l'a inventée, on emploie cette métaphore dans beaucoup de langues (en anglais, tissue, en allemand, Gewebe, en grec, istos - d'où l'histologie, la science qui étudie les tissus moléculaires...). Fleury semble prendre acte de cette situation et en tirer les conséquences ultimes. Si au départ, dans l'histoire des sciences, l'artefactuel a permis d'éclairer le naturel (le tissu textile permet de penser le tissu biologique), dans cette perspective, le naturel guidera l'artefactuel (le tissu biologique sert de modèle au tissu artefactuel).

Cette hypothèse rappelle les thèses fameuses de Gottfried Semper sur l'art textile, plus d'un siècle plus tôt. Même si Semper, d'un côté, pensait que seul l'homme se pare, d'un autre il cherchait à trouver les lois communes qui expliquent la formation des formes artefactuelles et des formes naturelles, s'inspirant de Goethe. Or l'art textile est un excellent objet d'étude pour déterminer ces lois. En effet son origine naturelle était clairement établie aux yeux de Semper : ce sont les treillis, les entrelacements de branches qui ont donné l'idée du tressage destiné à établir des barrières et des protections aux habitats (Wand, le mur). Ensuite, le procédé s'est développé en tissage et s'est appliqué aux vêtements (Gewand), puis à toute forme de revêtement et de décoration (Semper 2007 [1860] : 330-31). Si Semper avait pensé au caractère tissé de la peau, il aurait sans doute étendu son hypothèse au domaine animal. Il aurait pu ainsi montrer efficacement l'affinité profonde de la parure comme revêtement du corps avec la nature même du vivant.

Classiquement, les signaux visuels qu'envoie la modification artificielle de l'apparence humaine étaient interprétés comme une artificialisation de l'humain, son entrée dans la culture et son rejet de la nature. À partir du moment où l'on n'associe plus automatiquement parure et culture, culture et humanité, mais où l'on peut analyser morphogénétiquement la formation 
conjointe des apparences naturelles et artificielles, ces signaux peuvent être aussi interprétés comme une façon d'intensifier la vitalité de l'être humain. Dans une telle perspective, un être vivant en bonne santé aura les couleurs plus vives, les poils plus soyeux, le teint plus éclatant ; bref, il sera « en pleine forme ». C'est l'aspect organique de la parure qui est ici à l'honneur. Celle-ci «fait être plus », comme dit à peu près Simmel (Simmel $1998: 81$; et Flügel $1982: 26$ ).

L'animisme interprété par Descola nous avait servi à dépasser l'association hommeculture et animal-nature et à penser à la fois une parure portée par les animaux, comme des hommes. Pourtant, par le biais de l'héraldique, on a perçu de quelle façon l'apparence animiste était encore pensée sur un mode identitaire, sémiotique. Mais l'éthologie et la morphogenèse, sciences «naturalistes », remettent également en cause ce postulat, et ceci au moins de deux façons. Soit que le domaine de la culture est étendu au monde animal (ethnographie animale) - ce qui ouvre la possibilité d'une sémiotique des apparences animales. Soit que le domaine de la nature est étendu au monde humain (éthologie humaine). Alors la sémiotique des apparences est dépassée ; alors, véritablement, on entre dans un nouveau régime de pensée où la parure ne produit pas un langage qui se plaquerait sur le corps de l'animal comme sur la robe du seigneur, c'est-à-dire de l'extérieur, mais où l'on comprendrait que la parure manifeste une « poussée » vitale de l'intérieur. Dès lors, si l'on veut comprendre l'immense variété des formes de parures utilisées par les hommes sans passer par la codicologie du blason, c'est aux enseignements de la morphogenèse du vivant qu'il faut s'intéresser. Mais, dans cette perspective, tout ou presque reste à faire.

\section{Références bibliographiques}

Bartholeyns, G., 2008. Naissance d'une culture des apparences. Le vêtement en Occident, XIII'-XIVe siècle, thèse de doctorat, Université Libre de Bruxelles/EHESS, Paris, 2 vol.

2009. «L'Homme au risque du vêtement. Un indice d'humanité dans la culture occidentale», in G. Bartholeyns, P.-O. Dittmar, T. Golsenne, V. Jolivet, M. Har-Peled (éd.), Adam et l'astragale. Essais d'anthropologie et d'histoire sur les limites de l'humain. Paris : Éd. de la MSH, 99-136.

2011 (à paraître). «Le moment chrétien. Fondation antique de la culture vestimentaire médiévale », in F. Gherchanoc et V. Huet (éd.), Vêtements antiques. S'habiller, se déshabiller dans les mondes anciens, Paris.

Crouzet-Pavan, E., 2007. Renaissances italiennes. 1380-1500. Paris : Albin Michel, " Bibliothèque Histoire ».

Descola, Ph., 2005. Par-delà nature et culture. Paris : Gallimard, « Bibliothèque des sciences humaines ».

-_--, 2010. « Un monde animé », in Ph. Descola (éd.), La Fabrique des images. Visions du monde et formes de la représentation. Paris : Somogy-Musée du quai Branly, 23-38.

Dewitte, J., 1999. «Une comparaison fondamentale », in P. Robert (éd.), Animalité et humanité. Autour d'Adolf Portmann, $\mathrm{XV}$ e colloque annuel du groupe d'étude «Pratiques Sociales et Théories », Revue européenne des sciences sociales, 37 (115) : 9-31.

Fleury, V., 2005 [2003]. Des pieds et des mains. Genèse des formes de la nature. Paris : Flammarion, « Champs ».

Flügel, J. C., 1982 [1930]. Le rêveur nu. De la parure vestimentaire, trad. fr. de J.-M. Denis. Paris : AubierMontaigne, « La psychanalyse prise au mot».

Gell, A., 2009 [1998]. L'art et ses agents. Une théorie anthropologique, trad. fr. S. et O. Revault. Dijon : Les presses du réel, « Fabula ».

Golsenne, T., 2009. "L'ornement est-il animiste? », in A.-C. Taylor et T. Dufrêne (éd.), Cannibalismes disciplinaires. Quand l'histoire de l'art et l'anthropologie se rencontrent. Paris : INHA-Musée du quai Branly, 255-68. 
Gudin, C., 2003. Une histoire naturelle de la séduction. Paris : Seuil, « Science ouverte ».

Huergueta, S., 2005. «Vision - vision et évolution animale », in Encyclopadia Universalis, DVD-ROM, version 11. Paris : Opti Media.

Huizinga, J., 1967 [1919]. Le Déclin du Moyen Âge, trad. fr. J. Bastin. Paris : Payot, « Petite bibliothèque ».

LAgEt P., 2005. Nerveux (système) - Neurogenèse, in Encyclopcedia Universalis, DVD-ROM, version 11. Paris : Opti Media.

Leroi-Gourhan, A., 1965. Le Geste et la Parole. 2. La Mémoire et les Rythmes. Paris : Albin Michel, « Sciences d'aujourd'hui ».

Lestel D., 2009 [2001]. Les Origines animales de la culture. Paris : Flammarion, « Champs essais ».

LÉvi-STrauss, C., 1955. Tristes tropiques. Paris : Plon.

--_-, 1962. Le Totémisme aujourd'hui. Paris : PUF.

1985 [1958]. Anthropologie structurale. Paris : Pocket, « Agora ».

MAdDEn, J. et al., 2004. Local traditions of bower decoration by spotted bowerbirds in a single population, Animal Behaviour, $68: 759-65$.

Pastoureau, M., 1986. Figures et couleurs. Étude sur la symbolique et la sensibilité médiévales. Paris : Le Léopard d'Or.

PAstré, J.-M., 1994. «Les survivances d'un mythe: quelques images du guerrier-fauve dans la littérature allemande médiévale », in A. Niderst (éd.), L’Animalité. Hommes et animaux dans la littérature française. Tübingen : Gunter Narr Verlag, 67-76.

Perl-Treves, D., 1998. « Les cristaux du monde vivant », La Recherche, 305 : 34-38.

Ретітот, J., 2004. Morphologie et Esthétique. La Forme et le Sens chez Goethe, Lessing, Lévi-Strauss, Kant, Valéry, Husserl, Eco, Proust, Stendhal. Paris : Maisonneuve \& Larose, « Dynamiques du Sens ».

Portmann, A., 1961 [1948]. La Forme animale, trad. fr. de G. Remy. Paris : Payot.

Prévost, B., 2009. L'élégance animale. Esthétique et zoologie selon Adolf Portmann, Images re-vues, 6, 2009, http://www.imagesrevues.org/Article_Archive.php?id_article=40.

Rosset, C., 2004. L'Anti-nature. Eléments pour une philosophie tragique. Paris : PUF, « Quadrige ».

SEMPER, G., 2007. Du style et de l'architecture. Ecrits, 1834-1869, trad. fr. de J. Soulillou. Marseille : Parenthèses, «Eupalinos ».

Simmel, G., 1998. La parure et autres essais, trad. fr. et présentation de M. Collomb, P. Marty et F. Vinas. Paris : Éd. de la MSH, «Philia ».

Stamm, Rodger Alfred, 1999. «L'intériorité, dimension fondamentale de la vie », Animalité et humanité. Autour d'Adolf Portmann. Revue européenne des sciences sociales, 37 (115) : 57-73.

TABORIN, Y., 2004. Langage sans parole. La parure aux temps préhistoriques. Paris : La maison des roches.

TAYloR, A.-C., 2010. « Voir comme un Autre : figurations amazoniennes de l'âme et des corps », in Ph. Descola (éd.), La Fabrique des images. Visions du monde et formes de la représentation. Paris : Somogy-Musée du quai Branly, 41-50.

Tylor, E. B., 1920 [1871]. Primitive Culture, 2 vol. Londres : John Murray.

VIDAL, J.-M., 1987. "L'en-deçà du "stade du miroir": nature et culture de la pudeur et de la parure », Communications, $46: 7-29$.

VON DEN Steinen, K., 2005 [1925]. Les Marquisiens et leur art. L'ornementation primitive des mers du Sud, I : tatouage, trad. fr., s. 1. : Édition française, Musée de Tahiti et des îles-Te Fare Iamanaha.

von UexkÜLl, J., 1984 [1956]. Mondes animaux et monde humain, trad. fr. et présentation de P. Muller. Paris : Denoël, « Bibliothèque Médiations ». 


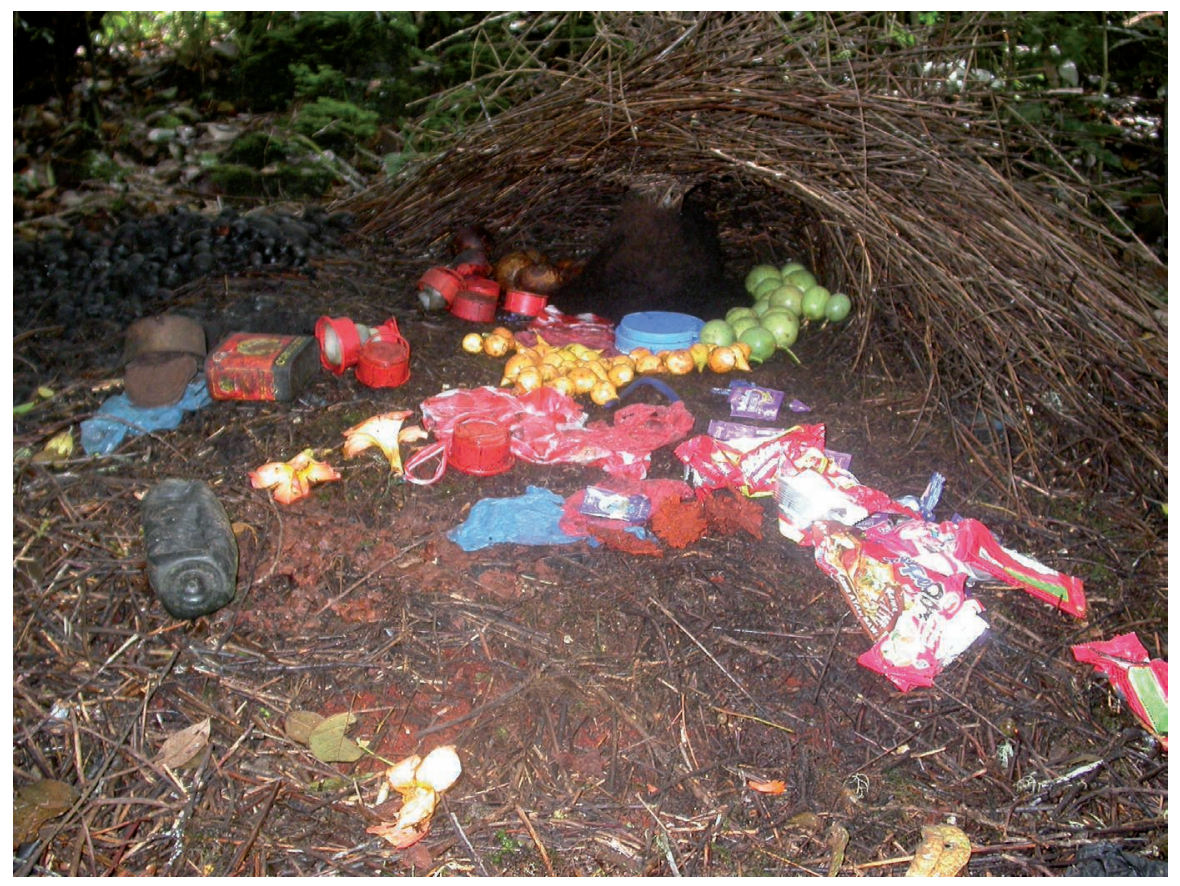

\section{Cf. Golsenne, figure 1}

Nid de grand jardinier, Papouasie occidentale, Arfak Mountains, 13 août 2008.

(C) Photo Collaert Brothers. 